\title{
Algorithms for treating equilibrium and fixed point problems
}

\author{
Xiaolong Qin', Sun Young Cho² and Lin Wang ${ }^{3 *}$
}

"Correspondence:

wl64mail@aliyun.com

${ }^{3}$ College of Statistics and

Mathematics, Yunnan University of

Finance and Economics, Kunming, 650221, China

Full list of author information is

available at the end of the article

\begin{abstract}
In this paper, a common solution problem is investigated based on a projection algorithm. Strong convergence theorems for common solutions of a system of equilibrium problems and a family of asymptotically quasi- $\phi$-nonexpansive mappings are established in a uniformly smooth and strictly convex Banach space which also enjoys the Kadec-Klee property.
\end{abstract}

Keywords: asymptotically quasi- $\phi$-nonexpansive mapping; equilibrium problem; fixed point; generalized projection; zero point

\section{Introduction}

Bifunction equilibrium problems, which include many important problems in nonlinear analysis and optimization, such as the Nash equilibrium problem, variational inequalities, complementarity problems, vector optimization problems, fixed point problems, saddle point problems and game theory, recently have been studied as an effective and powerful tool for studying many real world problems which arise in economics, finance, image reconstruction, ecology, transportation, and network; see [1-23] and the references therein. The theory of fixed points as an important branch of functional analysis is a bridge between nonlinear functional analysis and optimization. Indeed, lots of problems arising in economics, engineering, and physics can be studied by fixed point techniques. The study of fixed point approximation algorithms for computing fixed points constitutes now a topic of intensive research efforts. Many well-known problems can be studied by using algorithms which are iterative in their nature. As an example, in computer tomography with limited data, each piece of information implies the existence of a convex set in which the required solution lies. The problem of finding a point in the intersection of these convex subsets is then of crucial interest, and it cannot be usually solved directly. Therefore, an iterative algorithm must be used to approximate such a point. The well known convex feasibility problem, which captures applications in various disciplines such as image restoration and radiation therapy treatment planning, is to find a point in the intersection of common fixed point sets of a family of nonlinear mappings. Krasnoselskii-Mann iteration, which is also known as a one-step iteration, is a classic algorithm to study fixed points of nonlinear operators. However, Krasnoselskii-Mann iteration only enjoys weak convergence for nonexpansive mappings; see [24] and the references therein. There are a lot of real world problems which exist in infinite dimension spaces. In such problems, strong convergence or norm convergence is often much more desirable than weak convergence.

(อ2013 Qin et al.: licensee Springer. This is an Open Access article distributed under the terms of the Creative Commons Attribution License (http://creativecommons.org/licenses/by/2.0), which permits unrestricted use, distribution, and reproduction in any medium, provided the original work is properly cited. 
To guarantee the strong convergence of Krasnoselskii-Mann iteration, many authors use different regularization methods. The projection technique which was first introduced by Haugazeau [25] has been considered for the approximation of fixed points of nonexpansive mappings. The advantage of projection methods is that strong convergence of iterative sequences can be guaranteed without any compact assumptions.

In this paper, we study a common solution problem based on a projection algorithm. Strong convergence theorems of common solutions are established in a uniformly smooth and strictly convex Banach space which also enjoys the Kadec-Klee property.

\section{Preliminaries}

Let $E$ be a real Banach space, $E^{*}$ be the dual space of $E$ and $C$ be a nonempty subset of $E$. Let $f$ be a bifunction from $C \times C$ to $\mathbb{R}$, where $\mathbb{R}$ denotes the set of real numbers. Recall the following equilibrium problem: find $\bar{x} \in C$ such that

$$
f(\bar{x} y) \geq 0, \quad \forall y \in C .
$$

We use $E P(f)$ to denote the solution set of equilibrium problem (2.1). That is,

$$
E P(f)=\{x \in C: f(x, y) \geq 0, \forall y \in C\} .
$$

Given a mapping $A: C \rightarrow E^{*}$, let

$$
f(x, y)=\langle A x, y-x\rangle, \quad \forall x, y \in C .
$$

Then $\bar{x} \in E P(f)$ iff $\bar{x}$ is a solution of the following variational inequality: find $\bar{x}$ such that

$$
\langle A \bar{x} y-\bar{x}\rangle \geq 0, \quad \forall y \in C .
$$

In order to study the solution of problem (2.1), we assume that $f$ satisfies the following conditions:

(A1) $f(x, x)=0, \forall x \in C$;

(A2) $f$ is monotone, i.e., $f(x, y)+f(y, x) \leq 0, \forall x, y \in C$;

(A3)

$$
\limsup _{t \downarrow 0} f(t z+(1-t) x, y) \leq f(x, y), \quad \forall x, y, z \in C ;
$$

(A4) for each $x \in C, y \mapsto f(x, y)$ is convex and weakly lower semi-continuous.

Recall that a Banach space $E$ is said to be strictly convex iff $\left\|\frac{x+y}{2}\right\|<1$ for all $x, y \in E$ with $\|x\|=\|y\|=1$ and $x \neq y$. It is said to be uniformly convex iff $\lim _{n \rightarrow \infty}\left\|x_{n}-y_{n}\right\|=0$ for any two sequences $\left\{x_{n}\right\}$ and $\left\{y_{n}\right\}$ in $E$ such that $\left\|x_{n}\right\|=\left\|y_{n}\right\|=1$ and $\lim _{n \rightarrow \infty}\left\|\frac{x_{n}+y_{n}}{2}\right\|=1$. Let $U_{E}=\{x \in E:\|x\|=1\}$ be the unit sphere of $E$. Then the Banach space $E$ is said to be smooth iff

$$
\lim _{t \rightarrow 0} \frac{\|x+t y\|-\|x\|}{t}
$$

exists for each $x, y \in U_{E}$. It is also said to be uniformly smooth iff the above limit is attained uniformly for $x, y \in U_{E}$. It is well known that if $E$ is uniformly smooth, then $J$ is uniformly 
norm-to-norm continuous on each bounded subset of $E$. It is also well known that $E$ is uniformly smooth if and only if $E^{*}$ is uniformly convex.

Recall that a Banach space $E$ enjoys the Kadec-Klee property if for any sequence $\left\{x_{n}\right\} \subset E$, and $x \in E$ with $x_{n} \rightarrow x$, and $\left\|x_{n}\right\| \rightarrow\|x\|$, then $\left\|x_{n}-x\right\| \rightarrow 0$ as $n \rightarrow \infty$. It is well known that if $E$ is a uniformly convex Banach space, then $E$ enjoys the Kadec-Klee property. Let $T$ : $C \rightarrow C$ be a mapping. In this paper, we use $F(T)$ to denote the fixed point set of $T . T$ is said to be closed if for any sequence $\left\{x_{n}\right\} \subset C$ such that $\lim _{n \rightarrow \infty} x_{n}=x_{0}$ and $\lim _{n \rightarrow \infty} T x_{n}=y_{0}$, then $T x_{0}=y_{0}$. In this paper, we use $\rightarrow$ and $\rightarrow$ to denote strong convergence and weak convergence, respectively.

Recall that the normalized duality mapping $J$ from $E$ to $2^{E^{*}}$ is defined by

$$
J x=\left\{f^{*} \in E^{*}:\left\langle x, f^{*}\right\rangle=\|x\|^{2}=\left\|f^{*}\right\|^{2}\right\},
$$

where $\langle\cdot, \cdot\rangle$ denotes the generalized duality pairing. Next, we assume that $E$ is a smooth Banach space. Consider the functional defined by

$$
\phi(x, y)=\|x\|^{2}-2\langle x, J y\rangle+\|y\|^{2}, \quad \forall x, y \in E .
$$

Observe that, in a Hilbert space $H$, the equality is reduced to $\phi(x, y)=\|x-y\|^{2}, x, y \in H$. As we all know, if $C$ is a nonempty closed convex subset of a Hilbert space $H$ and $P_{C}: H \rightarrow C$ is the metric projection of $H$ onto $C$, then $P_{C}$ is nonexpansive. This fact actually characterizes Hilbert spaces and, consequently, it is not available in more general Banach spaces. In this connection, Alber [26] recently introduced a generalized projection operator $\Pi_{C}$ in a Banach space $E$ which is an analogue of the metric projection $P_{C}$ in Hilbert spaces. Recall that the generalized projection $\Pi_{C}: E \rightarrow C$ is a map that assigns to an arbitrary point $x \in E$ the minimum point of the functional $\phi(x, y)$, that is, $\Pi_{C} x=\bar{x}$, where $\bar{x}$ is the solution to the minimization problem

$$
\phi(\bar{x}, x)=\min _{y \in C} \phi(y, x)
$$

Existence and uniqueness of the operator $\Pi_{C}$ follows from the properties of the functional $\phi(x, y)$ and strict monotonicity of the mapping $J$. In Hilbert spaces, $\Pi_{C}=P_{C}$. It is obvious from the definition of a function $\phi$ that

$$
(\|x\|-\|y\|)^{2} \leq \phi(x, y) \leq(\|y\|+\|x\|)^{2}, \quad \forall x, y \in E,
$$

and

$$
\phi(x, y)=\phi(x, z)+\phi(z, y)+2\langle x-z, J z-J y\rangle, \quad \forall x, y, z \in E .
$$

Remark 2.1 If $E$ is a reflexive, strictly convex, and smooth Banach space, then $\phi(x, y)=0$ if and only if $x=y$.

Recall that a point $p$ in $C$ is said to be an asymptotic fixed point of a mapping $T$ [27] iff $C$ contains a sequence $\left\{x_{n}\right\}$ which converges weakly to $p$ such that $\lim _{n \rightarrow \infty}\left\|x_{n}-T x_{n}\right\|=0$. The set of asymptotic fixed points of $T$ will be denoted by $\widetilde{F}(T)$. 
Recall that a mapping $T$ is said to be relatively nonexpansive iff

$$
\widetilde{F}(T)=F(T) \neq \emptyset, \quad \phi(p, T x) \leq \phi(p, x), \quad \forall x \in C, \forall p \in F(T) .
$$

Recall that a mapping $T$ is said to be relatively asymptotically nonexpansive iff

$$
\widetilde{F}(T)=F(T) \neq \emptyset, \quad \phi\left(p, T^{n} x\right) \leq\left(1+\mu_{n}\right) \phi(p, x), \quad \forall x \in C, \forall p \in F(T), \forall n \geq 1,
$$

where $\left\{\mu_{n}\right\} \subset[0, \infty)$ is a sequence such that $\mu_{n} \rightarrow 0$ as $n \rightarrow \infty$.

Remark 2.2 The class of relatively asymptotically nonexpansive mappings was considered in [28] and [29]; see the references therein.

Recall that a mapping $T$ is said to be quasi- $\phi$-nonexpansive iff

$$
F(T) \neq \emptyset, \quad \phi(p, T x) \leq \phi(p, x), \quad \forall x \in C, \forall p \in F(T) .
$$

Recall that a mapping $T$ is said to be asymptotically quasi- $\phi$-nonexpansive iff there exists a sequence $\left\{\mu_{n}\right\} \subset[0, \infty)$ with $\mu_{n} \rightarrow 0$ as $n \rightarrow \infty$ such that

$$
F(T) \neq \emptyset, \quad \phi\left(p, T^{n} x\right) \leq\left(1+\mu_{n}\right) \phi(p, x), \quad \forall x \in C, \forall p \in F(T), \forall n \geq 1 .
$$

Remark 2.3 The class of asymptotically quasi- $\phi$-nonexpansive mappings was considered in Zhou et al. [30] and Qin et al. [31]; see also [32].

Remark 2.4 The class of quasi- $\phi$-nonexpansive mappings and the class of asymptotically quasi- $\phi$-nonexpansive mappings are more general than the class of relatively nonexpansive mappings and the class of relatively asymptotically nonexpansive mappings. Quasi$\phi$-nonexpansive mappings and asymptotically quasi- $\phi$-nonexpansive mappings do not require the restriction $F(T)=\widetilde{F}(T)$.

Remark 2.5 The class of quasi- $\phi$-nonexpansive mappings and the class of asymptotically quasi- $\phi$-nonexpansive mappings are generalizations of the class of quasi-nonexpansive mappings and the class of asymptotically quasi-nonexpansive mappings in Banach spaces.

In order to get our main results, we also need the following lemmas.

Lemma 2.6 [26] Let E be a reflexive, strictly convex, and smooth Banach space, let $C$ be a nonempty, closed, and convex subset of $E$, and let $x \in E$. Then

$$
\phi\left(y, \Pi_{C} x\right)+\phi\left(\Pi_{C} x, x\right) \leq \phi(y, x), \quad \forall y \in C .
$$

Lemma 2.7 [26] Let $C$ be a nonempty, closed, and convex subset of a smooth Banach space $E$, and let $x \in E$. Then $x_{0}=\Pi_{C} x$ if and only if

$$
\left\langle x_{0}-y, J x-J x_{0}\right\rangle \geq 0, \quad \forall y \in C
$$


Lemma 2.8 [33] Let E be a smooth and uniformly convex Banach space, and let $r>0$. Then there exists a strictly increasing, continuous, and convex function $g:[0,2 r] \rightarrow R$ such that $g(0)=0$ and

$$
\|t x+(1-t) y\|^{2} \leq t\|x\|^{2}+(1-t)\|y\|^{2}-t(1-t) g(\|x-y\|)
$$

for all $x, y \in B_{r}=\{x \in E:\|x\| \leq r\}$ and $t \in[0,1]$.

Lemma 2.9 Let $C$ be a closed convex subset of a smooth, strictly convex, and reflexive Banach space E. Let $f$ be a bifunction from $C \times C$ to $\mathbb{R}$ satisfying (A1)-(A4). Let $r>0$ and $x \in E$. Then

(a) [1] There exists $z \in C$ such that

$$
f(z, y)+\frac{1}{r}\langle y-z, J z-J x\rangle \geq 0, \quad \forall y \in C
$$

(b) [31,34] Define a mapping $T_{r}: E \rightarrow C$ by

$$
S_{r} x=\left\{z \in C: f(z, y)+\frac{1}{r}\langle y-z, J z-J x\rangle, \forall y \in C\right\} .
$$

Then the following conclusions hold:

(1) $S_{r}$ is single-valued;

(2) $S_{r}$ is a firmly nonexpansive-type mapping, i.e., for all $x, y \in E$,

$$
\left\langle S_{r} x-S_{r} y, J S_{r} x-J S_{r} y\right\rangle \leq\left\langle S_{r} x-S_{r} y, J x-J y\right\rangle
$$

(3) $F\left(S_{r}\right)=E P(f)$;

(4) $S_{r}$ is quasi- $\phi$-nonexpansive;

(5)

$$
\phi\left(q, S_{r} x\right)+\phi\left(S_{r} x, x\right) \leq \phi(q, x), \quad \forall q \in F\left(S_{r}\right)
$$

(6) $E P(f)$ is closed and convex.

Lemma 2.10 [35] Let E be a uniformly smooth and strictly convex Banach space which also enjoys the Kadec-Klee property. Let $C$ be a nonempty closed and convex subset of $E$. Let $T: C \rightarrow C$ be a closed asymptotically quasi- $\phi$-nonexpansive mapping. Then $F(T)$ is a closed convex subset of $C$.

\section{Main results}

Theorem 3.1 Let E be a uniformly smooth and strictly convex Banach space which also enjoys the Kadec-Klee property. Let $C$ be a nonempty closed and convex subset of E. Let $\Lambda$ be an index set. Let $f_{i}$ be a bifunction from $C \times C$ to $\mathbb{R}$ satisfying (A1)-(A4), and let $T_{i}: C \rightarrow C$ be an asymptotically quasi- $\phi$-nonexpansive mapping for every $i \in \Lambda$. Assume that $T_{i}$ is closed and uniformly asymptotically regular on $C$ for every $i \in \Lambda$. Assume that 
$\bigcap_{i \in \Lambda} F\left(T_{i}\right) \cap \bigcap_{i \in \Lambda} E F\left(f_{i}\right)$ is nonempty and bounded. Let $\left\{x_{n}\right\}$ be a sequence generated in the following manner:

$$
\left\{\begin{array}{l}
x_{0} \in E \quad \text { chosen arbitrarily, } \\
C_{(1, i)}=C, \\
C_{1}=\bigcap_{i \in \Lambda} C_{(1, i)}, \\
x_{1}=\Pi_{C_{1}} x_{0}, \\
y_{(n, i)}=J^{-1}\left(\alpha_{(n, i)} J x_{n}+\left(1-\alpha_{(n, i)}\right) J T_{i}^{n} x_{n}\right), \\
u_{(n, i)} \in C \text { such that } f_{i}\left(u_{(n, i)}, y\right)+\frac{1}{r_{(n, i)}}\left\langle y-u_{(n, i)}, J u_{(n, i)}-J y_{(n, i)}\right\rangle \geq 0, \quad \forall y \in C, \\
C_{(n+1, i)}=\left\{z \in C_{(n, i)}: \phi\left(z, u_{(n, i)}\right) \leq \phi\left(z, x_{n}\right)+\mu_{(n, i)} M_{(n, i)}\right\}, \\
C_{n+1}=\bigcap_{i \in \Lambda} C_{(n+1, i)}, \\
x_{n+1}=\Pi_{C_{n+1}} x_{1},
\end{array}\right.
$$

where $M_{(n, i)}=\sup \left\{\phi\left(p, x_{n}\right): p \in \bigcap_{i \in \Lambda} F\left(T_{i}\right) \cap \bigcap_{i \in \Lambda} E F\left(f_{i}\right)\right\},\left\{\alpha_{(n, i)}\right\}$ is a real sequence in $[0,1]$ such that $\liminf _{n \rightarrow \infty} \alpha_{(n, i)}\left(1-\alpha_{(n, i)}\right)>0$, and $\left\{r_{(n, i)}\right\}$ is a real sequence in $\left[a_{i}, \infty\right)$, where $\left\{a_{i}\right\}$ is a positive real number sequence, for every $i \in \Lambda$. Then the sequence $\left\{x_{n}\right\}$ converges strongly

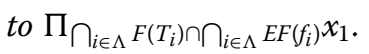

Proof We divide the proof into six steps.

Step 1. We prove that $\bigcap_{i \in \Lambda} F\left(T_{i}\right) \cap \bigcap_{i \in \Lambda} E F\left(f_{i}\right)$ is closed and convex.

In the light of Lemma 2.9 and 2.10, we easily find the conclusion.

Step 2. We prove that $C_{n}$ is closed and convex.

To show that $C_{n}$ is closed and convex, it suffices to show that for each fixed but arbitrary $i \in \Lambda, C_{(n, i)}$ is closed and convex. This can be proved by induction on $n$. It is obvious that $C_{(1, i)}=C$ is closed and convex. Assume that $C_{(k, i)}$ is closed and convex for some $k \geq 1$. For $z_{1}, z_{2} \in C_{(k+1, i)}$, we see that $z_{1}, z_{2} \in C_{(k, i)}$. It follows that $z=t z_{1}+(1-t) z_{2} \in C_{(k, i)}$, where $t \in(0,1)$. Notice that

$$
\phi\left(z_{1}, u_{(k, i)}\right) \leq \phi\left(z_{1}, x_{k}\right)+\mu_{(k, i)} M_{(k, i)}
$$

and

$$
\phi\left(z_{2}, u_{(k, i)}\right) \leq \phi\left(z_{2}, x_{k}\right)+\mu_{(k, i)} M_{(k, i)} .
$$

The above inequalities are equivalent to

$$
2\left\langle z_{1}, J x_{k}-J u_{(k, i)}\right\rangle \leq\left\|x_{k}\right\|^{2}-\left\|u_{(k, i)}\right\|^{2}+\mu_{(k, i)} M_{(k, i)}
$$

and

$$
2\left\langle z_{2}, J x_{k}-J u_{(k, i)}\right\rangle \leq\left\|x_{k}\right\|^{2}-\left\|u_{(k, i)}\right\|^{2}+\mu_{(k, i)} M_{(k, i)} .
$$

Multiplying $t$ and $(1-t)$ on the both sides of (3.1) and (3.2), respectively, yields that

$$
2\left\langle z, J x_{k}-J u_{(k, i)}\right\rangle \leq\left\|x_{k}\right\|^{2}-\left\|u_{(k, i)}\right\|^{2}+\mu_{(k, i)} M_{(k, i)} .
$$


That is,

$$
\phi\left(z, u_{(k, i)}\right) \leq \phi\left(z, x_{k}\right)+\mu_{(k, i)} M_{(k, i)},
$$

where $z \in C_{(k, i)}$. This finds that $C_{(k+1, i)}$ is closed and convex. We conclude that $C_{(n, i)}$ is closed and convex. This in turn implies that $C_{n}=\bigcap_{i \in \Lambda} C_{(n, i)}$ is closed and convex. This implies that $\Pi_{C_{n+1}} x_{1}$ is well defined.

Step 3. We prove that $\bigcap_{i \in \Lambda} F\left(T_{i}\right) \cap \bigcap_{i \in \Lambda} E F\left(f_{i}\right) \subset C_{n}$.

$\bigcap_{i \in \Lambda} F\left(T_{i}\right) \cap \bigcap_{i \in \Lambda} E F\left(f_{i}\right) \subset C_{1}=C$ is clear. Suppose that $\bigcap_{i \in \Lambda} F\left(T_{i}\right) \cap \bigcap_{i \in \Lambda} E F\left(f_{i}\right) \subset C_{(k, i)}$ for some positive integer $k$. For any $w \in \bigcap_{i \in \Lambda} F\left(T_{i}\right) \cap \bigcap_{i \in \Lambda} E F\left(f_{i}\right) \subset C_{(k, i)}$, we see that

$$
\begin{aligned}
\phi(w, & \left.u_{(k, i)}\right) \\
= & \phi\left(w, S_{r_{(k, i)}} y_{(k, i)}\right) \\
\leq & \phi\left(w, y_{(k, i)}\right) \\
= & \phi\left(w, J^{-1}\left(\alpha_{(k, i)} J x_{k}+\left(1-\alpha_{(k, i)}\right) J T_{i}^{k} x_{k}\right)\right) \\
= & \|w\|^{2}-2\left\langle w, \alpha_{(k, i)} J x_{k}+\left(1-\alpha_{(k, i)}\right) J T_{i}^{k} x_{k}\right\rangle \\
& +\left\|\alpha_{(k, i)} J x_{k}+\left(1-\alpha_{(k, i)}\right) J T_{i}^{k} x_{k}\right\|^{2} \\
\leq & \|w\|^{2}-2 \alpha_{(k, i)}\left\langle w, J x_{k}\right\rangle-2\left(1-\alpha_{(k, i)}\right)\left\langle w, J T_{i}^{k} x_{k}\right\rangle \\
& +\alpha_{(k, i)}\left\|x_{k}\right\|^{2}+\left(1-\alpha_{(k, i)}\right)\left\|T_{i}^{k} x_{k}\right\|^{2} \\
= & \alpha_{(k, i)} \phi\left(w, x_{k}\right)+\left(1-\alpha_{(k, i)}\right) \phi\left(w, T_{i}^{k} x_{k}\right) \\
\leq & \alpha_{(k, i)} \phi\left(w, x_{k}\right)+\left(1-\alpha_{(k, i)}\right) \phi\left(w, x_{k}\right)+\left(1-\alpha_{(k, i)}\right) \mu_{(k, i)} \phi\left(w, x_{k}\right) \\
\leq & \phi\left(w, x_{k}\right)+\mu_{(k, i)} \phi\left(w, x_{k}\right),
\end{aligned}
$$

which shows that $w \in C_{(k+1, i)}$. This implies that $\bigcap_{i \in \Lambda} F\left(T_{i}\right) \cap \bigcap_{i \in \Lambda} E F\left(f_{i}\right) \subset C_{(n, i)}$. This in turn implies that $\bigcap_{i \in \Lambda} F\left(T_{i}\right) \cap \bigcap_{i \in \Lambda} E F\left(f_{i}\right) \subset \bigcap_{i \in \Lambda} C_{(n, i)}$. This completes the proof that $\bigcap_{i \in \Lambda} F\left(T_{i}\right) \cap \bigcap_{i \in \Lambda} E F\left(f_{i}\right) \subset C_{n}$.

Step 4 . We prove that the sequence $\left\{x_{n}\right\}$ is bounded.

In the light of the construction $x_{n}=\Pi_{C_{n}} x_{1}$, we find from Lemma 2.7 that $\left\langle x_{n}-z, J x_{1}-\right.$ $\left.J x_{n}\right\rangle \geq 0$ for any $z \in C_{n}$.

Since $\bigcap_{i \in \Lambda} F\left(T_{i}\right) \cap \bigcap_{i \in \Lambda} E F\left(f_{i}\right) \subset C_{n}$, we find that

$$
\left\langle x_{n}-w, J x_{1}-J x_{n}\right\rangle \geq 0, \quad \forall w \in \bigcap_{i \in \Lambda} F\left(T_{i}\right) \cap \bigcap_{i \in \Lambda} E F\left(f_{i}\right) .
$$

On the other hand, we find from Lemma 2.6 that

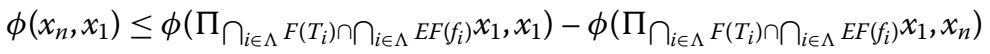

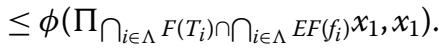

This implies that the sequence $\left\{\phi\left(x_{n}, x_{1}\right)\right\}$ is bounded. It follows from (2.3) that the sequence $\left\{x_{n}\right\}$ is also bounded. Since the space is reflexive, we may assume that $x_{n} \rightarrow \bar{x}$.

Step 5. We prove that $\bar{x} \in \bigcap_{i \in \Lambda} F\left(T_{i}\right) \cap \bigcap_{i \in \Lambda} E F\left(f_{i}\right)$. 
Since $C_{n}$ is closed and convex, we find that $\bar{x} \in C_{n}$. This implies that $\phi\left(x_{n}, x_{1}\right) \leq \phi\left(\bar{x}, x_{1}\right)$. On the other hand, we see from the weak lower semicontinuity of the norm that

$$
\begin{aligned}
\phi\left(\bar{x}, x_{1}\right) & =\|\bar{x}\|^{2}-2\left\langle\bar{x}, J x_{1}\right\rangle+\left\|x_{1}\right\|^{2} \\
& \leq \liminf _{n \rightarrow \infty}\left(\left\|x_{n}\right\|^{2}-2\left\langle x_{n}, J x_{1}\right\rangle+\left\|x_{1}\right\|^{2}\right) \\
& =\liminf _{n \rightarrow \infty} \phi\left(x_{n}, x_{1}\right) \\
& \leq \limsup _{n \rightarrow \infty} \phi\left(x_{n}, x_{1}\right) \\
& \leq \phi\left(\bar{x}, x_{1}\right),
\end{aligned}
$$

which implies that $\lim _{n \rightarrow \infty} \phi\left(x_{n}, x_{1}\right)=\phi\left(\bar{x}, x_{1}\right)$. Hence, we have $\lim _{n \rightarrow \infty}\left\|x_{n}\right\|=\|\bar{x}\|$. In view of the Kadec-Klee property of $E$, we find that $x_{n} \rightarrow \bar{x}$ as $n \rightarrow \infty$. Since $x_{n}=\Pi_{C_{n}} x_{1}$, and $x_{n+1}=\Pi_{C_{n+1}} x_{1} \in C_{n+1} \subset C_{n}$, we find that $\phi\left(x_{n}, x_{1}\right) \leq \phi\left(x_{n+1}, x_{1}\right)$. This shows that $\left\{\phi\left(x_{n}, x_{1}\right)\right\}$ is nondecreasing. We find from its boundedness that $\lim _{n \rightarrow \infty} \phi\left(x_{n}, x_{1}\right)$ exists. It follows that

$$
\begin{aligned}
\phi\left(x_{n+1}, x_{n}\right) & =\phi\left(x_{n+1}, \Pi_{C_{n}} x_{1}\right) \leq \phi\left(x_{n+1}, x_{1}\right)-\phi\left(\Pi_{C_{n}} x_{1}, x_{1}\right) \\
& =\phi\left(x_{n+1}, x_{1}\right)-\phi\left(x_{n}, x_{1}\right) .
\end{aligned}
$$

This implies that

$$
\lim _{n \rightarrow \infty} \phi\left(x_{n+1}, x_{n}\right)=0 .
$$

In the light of $x_{n+1}=\Pi_{C_{n+1}} x_{1} \in C_{n+1}$, we find that

$$
\phi\left(x_{n+1}, u_{(n, i)}\right) \leq \phi\left(x_{n+1}, x_{n}\right)+\mu_{(n, i)} M_{(n, i)} .
$$

This implies from (3.5) that

$$
\lim _{n \rightarrow \infty} \phi\left(x_{n+1}, u_{(n, i)}\right)=0 .
$$

In view of (2.3), we see that $\lim _{n \rightarrow \infty}\left(\left\|x_{n+1}\right\|-\left\|u_{(n, i)}\right\|\right)=0$. This implies that

$$
\lim _{n \rightarrow \infty}\left\|u_{(n, i)}\right\|=\|\bar{x}\|
$$

That is,

$$
\lim _{n \rightarrow \infty}\left\|J u_{(n, i)}\right\|=\lim _{n \rightarrow \infty}\left\|u_{(n, i)}\right\|=\|\bar{x}\|=\|J \bar{x}\| .
$$

This implies that $\left\{J u_{(n, i)}\right\}$ is bounded. Note that both $E$ and $E^{*}$ are reflexive. We may assume that $J u_{(n, i)} \rightarrow u^{(*, i)} \in E^{*}$. In view of the reflexivity of $E$, we see that $J(E)=E^{*}$. This shows that there exists an element $u^{i} \in E$ such that $J u^{i}=u^{(*, i)}$. It follows that

$$
\begin{aligned}
\phi\left(x_{n+1}, u_{(n, i)}\right) & =\left\|x_{n+1}\right\|^{2}-2\left\langle x_{n+1}, J u_{(n, i)}\right\rangle+\left\|u_{(n, i)}\right\|^{2} \\
& =\left\|x_{n+1}\right\|^{2}-2\left\langle x_{n+1}, J u_{(n, i)}\right\rangle+\left\|J u_{(n, i)}\right\|^{2} .
\end{aligned}
$$


Taking $\liminf f_{n \rightarrow \infty}$ on the both sides of the equality above from (3.5) yields that

$$
\begin{aligned}
0 & \geq\|\bar{x}\|^{2}-2\left\langle\bar{x}, u^{(*, i)}\right\rangle+\left\|u^{(*, i)}\right\|^{2} \\
& =\|\bar{x}\|^{2}-2\left\langle\bar{x}, J u^{i}\right\rangle+\left\|J u^{i}\right\|^{2} \\
& =\|\bar{x}\|^{2}-2\left\langle\bar{x}, J u^{i}\right\rangle+\left\|u^{i}\right\|^{2} \\
& =\phi\left(\bar{x}, u^{i}\right) .
\end{aligned}
$$

That is, $\bar{x}=u^{i}$, which in turn implies that $u^{(*, i)}=J \bar{x}$. It follows that $J u_{(n, i)} \rightarrow J \bar{x} \in E^{*}$. Since $E^{*}$ enjoys the Kadec-Klee property, we obtain from (3.7) that $\lim _{n \rightarrow \infty} J u_{(n, i)}=J \bar{x}$. Since $J^{-1}$ : $E^{*} \rightarrow E$ is demicontinuous and $E$ enjoys the Kadec-Klee property, we obtain that $u_{(n, i)} \rightarrow \bar{x}$, as $n \rightarrow \infty$. Note that $\left\|x_{n}-u_{(n, i)}\right\| \leq\left\|x_{n}-\bar{x}\right\|+\left\|\bar{x}-u_{(n, i)}\right\|$. It follows that

$$
\lim _{n \rightarrow \infty}\left\|x_{n}-u_{(n, i)}\right\|=0
$$

On the other hand, we have

$$
\begin{aligned}
\phi\left(w, x_{n}\right)-\phi\left(w, u_{(n, i)}\right) & =\left\|x_{n}\right\|^{2}-\left\|u_{(n, i)}\right\|^{2}-2\left\langle w, J x_{n}-J u_{(n, i)}\right\rangle \\
& \leq\left\|x_{n}-u_{(n, i)}\right\|\left(\left\|x_{n}\right\|+\left\|u_{(n, i)}\right\|\right)+2\|w\|\left\|J x_{n}-J u_{(n, i)}\right\| .
\end{aligned}
$$

In view of (3.8), we find that

$$
\lim _{n \rightarrow \infty}\left(\phi\left(w, x_{n}\right)-\phi\left(w, u_{(n, i)}\right)\right)=0 .
$$

Since $E$ is uniformly smooth, we know that $E^{*}$ is uniformly convex. In view of Lemma 2.8, we find that

$$
\begin{aligned}
& \phi\left(w, u_{(n, i)}\right) \\
&= \phi\left(w, S_{r_{(n, i)}} y_{(n, i)}\right) \\
& \leq \phi\left(w, y_{(n, i)}\right) \\
&= \phi\left(w, J^{-1}\left(\alpha_{(n, i)} J x_{n}+\left(1-\alpha_{(n, i)}\right) J T_{i}^{n} x_{n}\right)\right) \\
&=\|w\|^{2}-2\left\langle w, \alpha_{(n, i)} J x_{n}+\left(1-\alpha_{(n, i)}\right) J T_{i}^{n} x_{n}\right\rangle+\left\|\alpha_{(n, i)} J x_{n}+\left(1-\alpha_{(n, i)}\right) J T_{i}^{n} x_{n}\right\|^{2} \\
& \leq\|w\|^{2}-2 \alpha_{(n, i)}\left\langle w, J x_{n}\right\rangle-2\left(1-\alpha_{(n, i)}\right)\left\langle w, J T_{i}^{n} x_{n}\right\rangle \\
&+\alpha_{(n, i)}\left\|x_{n}\right\|^{2}+\left(1-\alpha_{(n, i)}\right)\left\|T_{i}^{n} x_{n}\right\|^{2}-\alpha_{(n, i)}\left(1-\alpha_{(n, i)}\right) g\left(\left\|J x_{n}-J T_{i}^{n} x_{n}\right\|\right) \\
&= \alpha_{(n, i)} \phi\left(w, x_{n}\right)+\left(1-\alpha_{(n, i)}\right) \phi\left(w, T_{i}^{n} x_{n}\right)-\alpha_{(n, i)}\left(1-\alpha_{(n, i)}\right) g\left(\left\|J x_{n}-J T_{i}^{n} x_{n}\right\|\right) \\
& \leq \phi\left(w, x_{n}\right)+\left(1-\alpha_{(n, i)}\right) \mu_{(n, i)} \phi\left(w, x_{n}\right)-\alpha_{(n, i)}\left(1-\alpha_{(n, i)}\right) g\left(\left\|J x_{n}-J T_{i}^{n} x_{n}\right\|\right) \\
& \leq \phi\left(w, x_{n}\right)+\mu \mu_{(n, i)} M_{(n, i)}-\alpha_{(n, i)}\left(1-\alpha_{(n, i)}\right) g\left(\left\|J x_{n}-J T_{i}^{n} x_{n}\right\|\right) .
\end{aligned}
$$

This implies that

$$
\alpha_{(n, i)}\left(1-\alpha_{(n, i)}\right) g\left(\left\|J x_{n}-J T_{i}^{n} x_{n}\right\|\right) \leq \phi\left(w, x_{n}\right)-\phi\left(w, u_{(n, i)}\right)+\mu_{(n, i)} M_{(n, i)} .
$$


In view of the restrictions on the sequence $\left\{\alpha_{(n, i)}\right\}$, we find from (3.9) that

$$
\lim _{n \rightarrow \infty}\left\|J x_{n}-J T_{i}^{n} x_{n}\right\|=0
$$

Notice that $\left\|J T_{i}^{n} x_{n}-J \bar{x}\right\| \leq\left\|J T_{i}^{n} x_{n}-J x_{n}\right\|+\left\|J x_{n}-J \bar{x}\right\|$. It follows that

$$
\lim _{n \rightarrow \infty}\left\|J T_{i}^{n} x_{n}-J \bar{x}\right\|=0
$$

The demicontinuity of $J^{-1}: E^{*} \rightarrow E$ implies that $T_{i}^{n} x_{n} \rightarrow \bar{x}$. Note that

$$
\left|\left\|T_{i}^{n} x_{n}\right\|-\|\bar{x}\|\right|=\left|\left\|J T_{i}^{n} x_{n}\right\|-\|J \bar{x}\|\right| \leq\left\|J T_{i}^{n} x_{n}-J \bar{x}\right\| .
$$

This implies from (3.10) that $\lim _{n \rightarrow \infty}\left\|T_{i}^{n} x_{n}\right\|=\|\bar{x}\|$. Since $E$ has the Kadec-Klee property, we obtain that $\lim _{n \rightarrow \infty}\left\|T_{i}^{n} x_{n}-\bar{x}\right\|=0$. On the other hand, we have

$$
\left\|T_{i}^{n+1} x_{n}-\bar{x}\right\| \leq\left\|T_{i}^{n+1} x_{n}-T_{i}^{n} x_{n}\right\|+\left\|T_{i}^{n} x_{n}-\bar{x}\right\|
$$

It follows from the uniformly asymptotic regularity of $T_{i}$ that

$$
\lim _{n \rightarrow \infty}\left\|T_{i}^{n+1} x_{n}-\bar{x}\right\|=0
$$

That is, $T_{i} T_{i}^{n} x_{n} \rightarrow \bar{x}$. From the closedness of $T_{i}$, we find $\bar{x}=T_{i} \bar{x}$ for each $i \in \Lambda$. This proves $\bar{x} \in \bigcap_{i \in \Lambda} F\left(T_{i}\right)$. Next, we show that $\bar{x} \in \bigcap_{i \in \Lambda} E F\left(f_{i}\right)$. In view of Lemma 2.6, we find that

$$
\begin{aligned}
\phi\left(u_{(n, i)}, y_{(n, i)}\right) & \leq \phi\left(w, y_{(n, i)}\right)-\phi\left(w, u_{(n, i)}\right) \\
& \leq \phi\left(w, x_{n}\right)+\mu_{(n, i)} \phi\left(w, x_{n}\right)-\phi\left(w, u_{(n, i)}\right) .
\end{aligned}
$$

It follows from (3.9) that $\lim _{n \rightarrow \infty} \phi\left(u_{(n, i)}, y_{(n, i)}\right)=0$. This implies that $\lim _{n \rightarrow \infty}\left(\left\|u_{(n, i)}\right\|-\right.$ $\left.\left\|y_{(n, i)}\right\|\right)=0$. In view of (3.8), we see that $u_{(n, i)} \rightarrow \bar{x}$ as $n \rightarrow \infty$. This implies that $\left\|y_{(n, i)}\right\|-$ $\|\bar{x}\| \rightarrow 0$, as $n \rightarrow \infty$. It follows that $\lim _{n \rightarrow \infty}\left\|J y_{(n, i)}\right\|=\|J \bar{x}\|$. This shows that $\left\{J y_{(n, i)}\right\}$ is bounded. Since $E^{*}$ is reflexive, we may assume that $J y_{(n, i)} \rightarrow y^{(*, i)} \in E^{*}$. In view of $J(E)=E^{*}$, we see that there exists $y^{i} \in E$ such that $J y^{i}=y^{(*, i)}$. It follows that

$$
\begin{aligned}
\phi\left(u_{(n, i)}, y_{(n, i)}\right) & =\left\|u_{(n, i)}\right\|^{2}-2\left\langle u_{(n, i)}, J y_{(n, i)}\right\rangle+\left\|y_{(n, i)}\right\|^{2} \\
& =\left\|u_{(n, i)}\right\|^{2}-2\left\langle u_{(n, i)}, J y_{(n, i)}\right\rangle+\left\|J y_{(n, i)}\right\|^{2} .
\end{aligned}
$$

Taking $\liminf _{n \rightarrow \infty}$ on the both sides of the equality above yields that

$$
\begin{aligned}
0 & \geq\|\bar{x}\|^{2}-2\left\langle\bar{x}, y^{(*, i)}\right\rangle+\left\|y^{(*, i)}\right\|^{2} \\
& =\|\bar{x}\|^{2}-2\left\langle\bar{x}, J y^{i}\right\rangle+\left\|J y^{i}\right\|^{2} \\
& =\|\bar{x}\|^{2}-2\left\langle\bar{x}, J y^{i}\right\rangle+\left\|y^{i}\right\|^{2} \\
& =\phi\left(\bar{x}, y^{i}\right) .
\end{aligned}
$$

That is, $\bar{x}=y^{i}$, which in turn implies that $y^{(*, i)}=J \bar{x}$. It follows that $J y_{(n, i)} \rightarrow J \bar{x} \in E^{*}$. Since $E^{*}$ enjoys the Kadec-Klee property, we obtain that $J y_{(n, i)}-J \bar{x} \rightarrow 0$ as $n \rightarrow \infty$. Note that 
$J^{-1}: E^{*} \rightarrow E$ is demicontinuous. It follows that $y_{(n, i)} \rightarrow \bar{x}$. Since $E$ enjoys the Kadec-Klee property, we obtain that $y_{(n, i)} \rightarrow \bar{x}$ as $n \rightarrow \infty$. Note that

$$
\left\|u_{(n, i)}-y_{(n, i)}\right\| \leq\left\|u_{(n, i)}-\bar{x}\right\|+\left\|\bar{x}-y_{(n, i)}\right\| .
$$

This implies that

$$
\lim _{n \rightarrow \infty}\left\|u_{(n, i)}-y_{(n, i)}\right\|=0 .
$$

Since $J$ is uniformly norm-to-norm continuous on any bounded sets, we have

$$
\lim _{n \rightarrow \infty}\left\|J u_{(n, i)}-J y_{(n, i)}\right\|=0 .
$$

From the assumption $r_{(n, i)} \geq a_{i}$, we see that

$$
\lim _{n \rightarrow \infty} \frac{\left\|J u_{(n, i)}-J y_{(n, i)}\right\|}{r_{(n, i)}}=0 .
$$

In view of $u_{(n, i)}=S_{r_{(n, i)}} y_{(n, i)}$, we see that

$$
f_{i}\left(u_{(n, i)}, y\right)+\frac{1}{r_{(n, i)}}\left\langle y-u_{(n, i)}, J u_{(n, i)}-J y_{(n, i)}\right\rangle \geq 0, \quad \forall y \in C .
$$

It follows from (A2) that

$$
\begin{aligned}
\left\|y-u_{(n, i)}\right\| \frac{\left\|J u_{(n, i)}-J y_{(n, i)}\right\|}{r_{n}} & \geq \frac{1}{r_{(n, i)}}\left\langle y-u_{(n, i)}, J u_{(n, i)}-J y_{(n, i)}\right\rangle \\
& \geq f_{i}\left(y, u_{(n, i)}\right), \quad \forall y \in C .
\end{aligned}
$$

In view of (A4), we find from (3.11) that

$$
f_{i}(y, \bar{x}) \leq 0, \quad \forall y \in C
$$

For $0<t_{i}<1$ and $y \in C$, define $y_{(t, i)}=t_{i} y+\left(1-t_{i}\right) \bar{x}$. It follows that $y_{(t, i)} \in C$, which yields that $f_{i}\left(y_{(t, i)}, \bar{x}\right) \leq 0$. It follows from the (A1) and (A4) that

$$
0=f_{i}\left(y_{(t, i)}, y_{(t, i)}\right) \leq t_{i} f_{i}\left(y_{(t, i)}, y\right)+\left(1-t_{i}\right) f_{i}\left(y_{(t, i)}, \bar{x}\right) \leq t_{i} f_{i}\left(y_{(t, i)}, y\right) .
$$

That is,

$$
f_{i}\left(y_{(t, i)}, y\right) \geq 0 .
$$

Letting $t_{i} \downarrow 0$, we obtain from (A3) that $f_{i}(\bar{x}, y) \geq 0, \forall y \in C$. This implies that $\bar{x} \in E P\left(f_{i}\right)$ for every $i \in \Lambda$. This shows that $\bar{x} \in \bigcap_{i \in \Lambda} E F\left(f_{i}\right)$. This completes the proof that $\bar{x} \in$ $\bigcap_{i \in \Lambda} F\left(T_{i}\right) \cap \bigcap_{i \in \Lambda} E F\left(f_{i}\right)$.

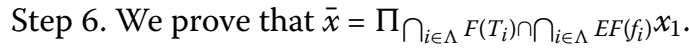


Letting $n \rightarrow \infty$ in (3.4), we see that

$$
\left\langle\bar{x}-w, J x_{1}-J \bar{x}\right\rangle \geq 0, \quad \forall w \in C S S
$$

In view of Lemma 2.7, we find that $\bar{x}=\Pi_{\bigcap_{i \in \Lambda} F\left(T_{i}\right) \cap \bigcap_{i \in \Lambda} E F\left(f_{i}\right)} x_{1}$. This completes the proof.

Remark 3.2 Theorem 3.1 mainly improves Theorem 2.1 of Qin et al. [29] in the following aspects:

(1) improves the mappings from a finite family of mappings to an infinite family of mappings

(2) extends the framework of the space from a uniformly smooth and uniformly convex space to a uniformly smooth and strictly convex Banach space which also enjoys the Kadec-Klee property.

If $T$ is a quasi- $\phi$-nonexpansive mapping, we find from Theorem 2.1 the following.

Corollary 3.3 Let E be a uniformly smooth and strictly convex Banach space which also enjoys the Kadec-Klee property. Let $C$ be a nonempty closed and convex subset of E. Let $\Lambda$ be an index set. Let $f_{i}$ be a bifunction from $C \times C$ to $\mathbb{R}$ satisfying (A1)-(A4), and let $T_{i}: C \rightarrow$ $E$ be a closed quasi- $\phi$-nonexpansive mapping for every $i \in \Lambda$. Assume that $\bigcap_{i \in \Lambda} F\left(T_{i}\right) \cap$ $\bigcap_{i \in \Lambda} E F\left(f_{i}\right)$ is nonempty. Let $\left\{x_{n}\right\}$ be a sequence generated in the following manner:

$$
\left\{\begin{array}{l}
x_{0} \in E \quad \text { chosen arbitrarily, } \\
C_{(1, i)}=C, \\
C_{1}=\bigcap_{i \in \Lambda} C_{(1, i)}, \\
x_{1}=\Pi_{C_{1}} x_{0}, \\
y_{(n, i)}=J^{-1}\left(\alpha_{(n, i)} J x_{n}+\left(1-\alpha_{(n, i)}\right) J T_{i} x_{n}\right), \\
u_{(n, i)} \in C \text { such that } f_{i}\left(u_{(n, i)}, y\right)+\frac{1}{r_{(n, i)}}\left\langle y-u_{(n, i)}, J u_{(n, i)}-J y_{(n, i)}\right\rangle \geq 0, \quad \forall y \in C, \\
C_{(n+1, i)}=\left\{z \in C_{(n, i)}: \phi\left(z, u_{(n, i)}\right) \leq \phi\left(z, x_{n}\right)\right\}, \\
C_{n+1}=\bigcap_{i \in \Lambda} C_{(n+1, i)}, \\
x_{n+1}=\Pi_{C_{n+1}} x_{1},
\end{array}\right.
$$

where $\left\{\alpha_{(n, i)}\right\}$ is a real sequence in $[0,1]$ such that $\liminf _{n \rightarrow \infty} \alpha_{(n, i)}\left(1-\alpha_{(n, i)}\right)>0$, and $\left\{r_{(n, i)}\right\}$ is a real sequence in $\left[a_{i}, \infty\right)$, where $\left\{a_{i}\right\}$ is a positive real number sequence for every $i \in \Lambda$. Then the sequence $\left\{x_{n}\right\}$ converges strongly to $\prod_{\bigcap_{i \in \Lambda} F\left(T_{i}\right) \cap \bigcap_{i \in \Lambda} E F\left(f_{i}\right)} x_{1}$.

Remark 3.4 Corollary 3.3 can be viewed as an extension of the corresponding results announced in [36] and [37].

Since every uniformly convex Banach space is a strictly convex Banach space which also enjoys the Kadec-Klee property, we see that Theorem 3.1 is still valid in a uniformly smooth and uniformly convex Banach space. Theorem 3.1 improves the corresponding results in Qin et al. [31].

For a single bifunction and mapping, we find from Theorem 3.1 the following. 
Corollary 3.5 Let E be a uniformly smooth and strictly convex Banach space which also enjoys the Kadec-Klee property. Let $C$ be a nonempty closed and convex subset of E. Let $f$ be a bifunction from $C \times C$ to $\mathbb{R}$ satisfying (A1)-(A4), and let $T: C \rightarrow E$ be an asymptotically quasi- $\phi$-nonexpansive mapping. Assume that $T$ is closed and uniformly asymptotically regular on $C$. Assume that $F(T) \cap E F(f)$ is nonempty. Let $\left\{x_{n}\right\}$ be a sequence generated in the following manner:

$$
\left\{\begin{array}{l}
x_{0} \in E \quad \text { chosen arbitrarily, } \\
C_{1}=C \\
x_{1}=\Pi_{C_{1}} x_{0}, \\
y_{n}=J^{-1}\left(\alpha_{n} J x_{n}+\left(1-\alpha_{n}\right) J T^{n} x_{n}\right), \\
u_{n} \in C \text { such that } f\left(u_{n}, y\right)+\frac{1}{r_{n}}\left\langle y-u_{n}, J u_{n}-J y_{n}\right\rangle \geq 0, \quad \forall y \in C, \\
C_{n+1}=\left\{z \in C_{n}: \phi\left(z, u_{n}\right) \leq \phi\left(z, x_{n}\right)+\mu_{n} M_{n}\right\}, \\
x_{n+1}=\Pi_{C_{n+1}} x_{1},
\end{array}\right.
$$

where $M_{n}=\sup \left\{\phi\left(p, x_{n}\right): p \in F(F) \cap E F(f)\right\},\left\{\alpha_{n}\right\}$ is a real sequence in $[0,1]$ such that $\liminf _{n \rightarrow \infty} \alpha_{n}\left(1-\alpha_{n}\right)>0$, and $\left\{r_{n}\right\}$ is a real sequence in $[a, \infty)$, where $a$ is a positive real number. Then the sequence $\left\{x_{n}\right\}$ converges strongly to $\Pi_{F(T) \cap E F(f)} x_{1}$.

If $T$ is the identity mapping, then Theorem 3.1 is reduced to the following.

Corollary 3.6 Let E be a uniformly smooth and strictly convex Banach space which also enjoys the Kadec-Klee property. Let $C$ be a nonempty closed and convex subset of E. Let $\Lambda$ be an index set. Let $f_{i}$ be a bifunction from $C \times C$ to $\mathbb{R}$ satisfying (A1)-(A4) for every $i \in \Lambda$. Assume that $\bigcap_{i \in \Lambda} E F\left(f_{i}\right)$ is nonempty and bounded. Let $\left\{x_{n}\right\}$ be a sequence generated in the following manner:

$$
\left\{\begin{array}{l}
x_{0} \in E \quad \text { chosen arbitrarily, } \\
C_{(1, i)}=C, \\
C_{1}=\bigcap_{i \in \Lambda} C_{(1, i)}, \\
x_{1}=\prod_{C_{1}} x_{0}, \\
u_{(n, i)} \in C \text { such that } f_{i}\left(u_{(n, i)}, y\right)+\frac{1}{r_{(n, i)}}\left\langle y-u_{(n, i)}, J u_{(n, i)}-J x_{n}\right\rangle \geq 0, \quad \forall y \in C, \\
C_{(n+1, i)}=\left\{z \in C_{(n, i)}: \phi\left(z, u_{(n, i)}\right) \leq \phi\left(z, x_{n}\right)+\mu_{(n, i)} M_{(n, i)}\right\}, \\
C_{n+1}=\bigcap_{i \in \Lambda} C_{(n+1, i)}, \\
x_{n+1}=\prod_{C_{n+1}} x_{1},
\end{array}\right.
$$

where $M_{(n, i)}=\sup \left\{\phi\left(p, x_{n}\right): p \in \bigcap_{i \in \Lambda} E F\left(f_{i}\right)\right\},\left\{\alpha_{(n, i)}\right\}$ is a real sequence in $[0,1]$ such that $\liminf _{n \rightarrow \infty} \alpha_{(n, i)}\left(1-\alpha_{(n, i)}\right)>0$, and $\left\{r_{(n, i)}\right\}$ is a real sequence in $\left[a_{i}, \infty\right)$, where $\left\{a_{i}\right\}$ is a positive real number sequence, for every $i \in \Lambda$. Then the sequence $\left\{x_{n}\right\}$ converges strongly to $\Pi_{\bigcap_{i \in \Lambda} E F\left(f_{i}\right)} x_{1}$. 
Authors' contributions

All authors contributed equally to this manuscript. All authors read and approved the final manuscript.

\section{Author details}

'Department of Mathematics, Hangzhou Normal University, Hangzhou, 310036, China. ${ }^{2}$ Department of Mathematics, Gyeongsang National University, Jinju, 660-701, Korea. ${ }^{3}$ College of Statistics and Mathematics, Yunnan University of Finance and Economics, Kunming, 650221, China.

\section{Acknowledgements}

The authors are grateful to the anonymous reviewers for useful suggestions which improved the contents of the article.

Received: 3 June 2013 Accepted: 31 October 2013 Published: 22 Nov 2013

\section{References}

1. Blum, E, Oettli, W: From optimization and variational inequalities to equilibrium problems. Math. Stud. 63, 123-145 (1994)

2. Gwinner, J, Raciti, F: Random equilibrium problems on networks. Math. Comput. Model. 43, 880-891 (2006)

3. Cho, SY, Li, W, Kang, SM: Convergence analysis of an iterative algorithm for monotone operators. J. Inequal. Appl. 2013, Article ID 199 (2013)

4. Osu, BO, Solomon, OU: A stochastic algorithm for the valuation of financial derivatives using the hyperbolic distributional variates. Math. Finance Lett. 1, 43-56 (2012)

5. Al-Bayati, AY, Al-Kawaz, RZ: A new hybrid WC-FR conjugate gradient-algorithm with modified secant condition for unconstrained optimization. J. Math. Comput. Sci. 2, 937-966 (2012)

6. Yuan, Q: Some results on asymptotically quasi- $\phi$-nonexpansive mappings in the intermediate sense. J. Fixed Point Theory 2012, Article ID 1 (2012)

7. Cho, SY, Qin, X, Kang, SM: Iterative processes for common fixed points of two different families of mappings with applications. J. Glob. Optim. 57, 1429-1446 (2013)

8. Dafermos, S, Nagurney, A: A network formulation of market equilibrium problems and variational inequalities. Oper. Res. Lett. 3, 247-250 (1984)

9. Tanaka, Y: A constructive version of Ky Fan's coincidence theorem. J. Math. Comput. Sci. 2, 926-936 (2012)

10. Cho, SY, Kang, SM: Approximation of fixed points of pseudocontraction semigroups based on a viscosity iterative process. Appl. Math. Lett. 24, 224-228 (2011)

11. Barbagallo, A: Existence and regularity of solutions to nonlinear degenerate evolutionary variational inequalities with applications to dynamic network equilibrium problems. Appl. Math. Comput. 208, 1-13 (2009)

12. Cho, SY, Kang, SM: Approximation of common solutions of variational inequalities via strict pseudocontractions. Acta Math. Sci. 32, 1607-1618 (2012)

13. Chen, JH: Iterations for equilibrium and fixed point problems. J. Nonlinear Funct. Anal. 2013, Article ID 4 (2013)

14. Carmi, A, Censor, Y, Gurfil, P: Convex feasibility modeling and projection methods for sparse signal recovery. J. Comput. Appl. Math. 236, 4318-4335 (2012)

15. He, R: Coincidence theorem and existence theorems of solutions for a system of Ky Fan type minimax inequalities in FC-spaces. Adv. Fixed Point Theory 2, 47-57 (2012)

16. Noor, MA, Noor, Kl, Waseem, M: Decompsition method for solving system of linear equations. Eng. Math. Lett. 2 34-41 (2013)

17. Shen, J, Pang, LP: An approximate bundle method for solving variational inequalities. Commun. Optim. Theory 1 $1-18(2012)$

18. Dhage, BC, Jadhav, NS: Differential inequalities and comparison theorems for first order hybrid integro-differential equations. Adv. Inequal. Appl. 2,61-80 (2013)

19. Zegeye, H, Shahzad, N: Strong convergence theorem for a common point of solution of variational inequality and fixed point problem. Adv. Fixed Point Theory 2, 374-397 (2012)

20. Park, S: A review of the KKM theory on $\phi_{A}$-space or GFC-spaces. Adv. Fixed Point Theory 3, 355-382 (2013)

21. Boonchari, D, Saejung, S: Approximation of common fixed point of a countable family of relatively nonexpansive mapping. Fixed Point Theory Appl. 2010, Article ID 407651 (2010)

22. Nilsrakoo, W, Saejung, S: On the fixed-point set of a family of relatively nonexpansive and generalized nonexpansive mappings. Fixed Point Theory Appl. 2010, Article ID 414232 (2010)

23. Saejung, S: Ray's theorem for firmly nonexpansive-like mappings and equilibrium problems in Banach spaces. Fixed Point Theory Appl. 2010, Article ID 806837 (2010)

24. Genel, A, Lindenstruss, J: An example concerning fixed points. Isr. J. Math. 22, 81-86 (1975)

25. Haugazeau, Y: Sur les inéquations variationnelles et la minimisation de fonctionnelles convexes. Ph.D. thesis, Université de Paris (1968)

26. Alber, Yal: Metric and generalized projection operators in Banach spaces: properties and applications. In: Kartsatos, AG (ed.) Theory and Applications of Nonlinear Operators of Accretive and Monotone Type. Dekker, New York (1996)

27. Reich, S: A weak convergence theorem for the alternating method with Bregman distance. In: Kartsatos, AG (ed.) Theory and Applications of Nonlinear Operators of Accretive and Monotone Type. Dekker, New York (1996)

28. Qin, X, Su, Y, Wu, C, Liu, K: Strong convergence theorems for nonlinear operators in Banach spaces. Commun. Appl. Nonlinear Anal. 14, 35-50 (2007)

29. Agarwal, RP, Cho, YJ, Qin, X: Generalized projection algorithms for nonlinear operators. Numer. Funct. Anal. Optim. 28 1197-1215 (2007)

30. Zhou, H, Gao, G, Tan, B: Convergence theorems of a modified hybrid algorithm for a family of quasi- $\boldsymbol{\phi}$-asymptotically nonexpansive mappings. SIAM J. Sci. Comput. 32, 453-464 (2010)

31. Qin, X, Cho, YJ, Kang, SM: Convergence theorems of common elements for equilibrium problems and fixed point problems in Banach spaces. J. Comput. Appl. Math. 225, 20-30 (2009)

32. Qin, X, Cho, SY, Kang, SM: On hybrid projection methods for asymptotically quasi- $\boldsymbol{\phi}$-nonexpansive mappings. Appl. Math. Comput. 215, 3874-3883 (2010) 
33. Zǎlinescu, C: On uniformly convex functions. J. Math. Anal. Appl. 95, 344-374 (1983)

34. Takahashi, W, Zembayashi, K: Strong and weak convergence theorems for equilibrium problems and relatively nonexpansive mappings in Banach spaces. Nonlinear Anal. 70, 45-57 (2009)

35. Qin, X, Huang, S, Wang, T: On the convergence of hybrid projection algorithms for asymptotically quasi- $\phi$-nonexpansive mappings. Comput. Math. Appl. 61, 851-859 (2011)

36. Qin, X, Cho, SY, Kang, SM: Strong convergence of shrinking projection methods for quasi- $\boldsymbol{\phi}$-nonexpansive mappings and equilibrium problems. J. Comput. Appl. Math. 234, 750-760 (2010)

37. Kim, JK: Strong convergence theorems by hybrid projection methods for equilibrium problems and fixed point problems of the asymptotically quasi- $\boldsymbol{\phi}$-nonexpansive mappings. Fixed Point Theory Appl. 2011, Article ID 10 (2011)

10.1186/1687-1812-2013-308

Cite this article as: Qin et al.: Algorithms for treating equilibrium and fixed point problems. Fixed Point Theory and Applications 2013, 2013:308

\section{Submit your manuscript to a SpringerOpen ${ }^{\circ}$ journal and benefit from:}

- Convenient online submission

- Rigorous peer review

- Immediate publication on acceptance

- Open access: articles freely available online

- High visibility within the field

- Retaining the copyright to your article 